\title{
A retrospective notes-based review of patients lost to follow-up from anti-retroviral therapy at Mulanje Mission Hospital, Malawi
}

Silas Webb ${ }^{1}$, Joseph Hartland ${ }^{2}$

1. Croydon University Hospital and University of Bristol

2. Great Western Hospital and University of Bristol

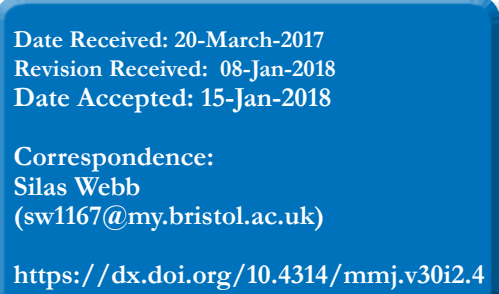

Date Received: 20-March-2017 Revision Received: 08-Jan-2018

(sw1167@my.bristol.ac.uk)

https://dx.doi.org/10.4314/mmj.v30i2.4

\begin{abstract}
Aim
Abstract

To analyse patients with HIV who were lost to follow-up from anti-retroviral therapy (ART) at Mulanje Mission Hospital (MMH), Malawi.

Methods

All patients on adult antiretroviral combinations at MMH, who were classified as lost to follow-up (LTFU) according to the national guidelines (patients missing a scheduled follow-up visit by more than two months) over a 12-month period, were included in the study and compared against a control group who had never been lost. Variables compared were gender, age, months on ART, time of year, WHO clinical stage, ART regimen, reported side effects, number of doses missed in the previous 12 months, whether the patient has been followed up in the community and if so, the length of time elapsed before follow-up.

Results

In all, 136 patients had been LTFU over the previous 12 months at MMH. Of these, 43 had incomplete or missing ART cards, resulting in 93 LTFU patient's data that could be analysed. Patients were more likely to get LTFU if they were men ( $p=0.03$ ), who had been on anti-retroviral therapy for a short duration $(\mathrm{p}=0.06)$ and the proportion of patients who missed more than 4 doses in the previous 12 months was higher among LTFU patients $(p=0.05)$. Only $34.4 \%$ of those LTFU had been traced in the community at the time of analysis. Of those traced, $27 \%$ had moved to another area, $5.5 \%$ had died, $5.5 \%$ had the wrong documentation and $62 \%$ gave no reason as to why they had missed appointments.

Conclusion

This study in $\mathrm{MMH}$ has highlighted the importance and feasibility of comprehensive facility-level data-collection, both to identify local patient populations at risk of becoming lost to follow-up and to assess the follow-up measures in place to bring these lost to follow-up patients back into the programme. Even in the short time and with the small sample that was collected, there was evidence that patients most likely to get LTFU in MMH were young men, who had been on anti-retroviral therapy for a short duration and had missed over 4 doses in the last 12 months.
\end{abstract}

\section{Introduction}

More than three decades after the first cases of Human Immunodeficiency Virus (HIV) infections were recognized, the epidemic in sub-Saharan Africa (SSA) has claimed the lives of over 36 million people, and is still one of the world's most important public health issues ${ }^{1}$. However, in recent years, significant advances have been made to address the epidemic, in line with the political intent of Millennium Development Goal 6, which was first to halve then begin to reverse the spread of HIV by $2015^{2}$. Key to this success has been the expansion of antiretroviral therapy (ART), which has led to a 30-fold increase in ART coverage in SSA over the last decade ${ }^{3}$.

For patients accessing ART, Acquired Immune Deficiency Syndrome (AIDS) has been transformed from a lifelimiting condition, with a median survival of one year from diagnosis, into a chronic disease ${ }^{4}$, one which requires lifelong management ${ }^{5}$. However, the long-term retention of patients on ART has, itself, become a major challenge in SSA. In 2010, a systematic review of 39 papers found that $30 \%$ of patients were no longer taking their ARTs two years after starting treatment ${ }^{6}$. In the same review, it was noted that there was a paucity of data as to why patients become lost to follow-up (LTFU) in $\mathrm{SSA}^{6}$.

This study will examine the current prevalence and reasons for patients becoming LTFU in resource-poor settings. The particular focus is on Mulanje Mission Hospital (MMH) in Malawi, serving one of the poorest regions of a nation with the lowest Gross Domestic Product (GDP) per capita in the world ${ }^{7}$.

\section{Definitions}

Throughout the literature, there is no universal definition for patients who are not retained in AR'T programmes. The World Health Organization (WHO) defines retention as "patients known to be alive and receiving highly active ART (HAART) at the end of a follow-up period." 8 This vague definition, which does not prescribe a specific follow-up period, has led to a wide disparity of retention periods being used in the studies: 30 days $^{9}, 3$ months $^{10}, 6$ months $^{11}$ and 1 year ${ }^{12}$ are all quoted. Further confusion arises from the term 'LTFU' being used interchangeably with 'attrition' and 'defaulting' across studies $^{5}$. In this report, LTFU has been utilized instead of 
the two latter terms, which have been criticized for assigning blame to the patients as opposed to "

\section{Prevalence of patients LTFU from ART in SSA}

The largest systematic review, which aimed to quantify the number of patients retained on ART in SSA (using 39 cohort studies with a total of 226,307 patients), found that retention was $70 \%$ after 24 months. Although this study brought substantial attention to the issue, it did not differentiate the three distinct cohorts of patients who were not retained in care. One study which looked at samples of patient Mat retained in ART across 13 countries in SSA, including and $4 \%$ were retained in care despite stopping ART $^{3}$. In 2008, a more detailed single-cohort study in South Africa found that $31 \%$ of their non-retained patients had died, $25 \%$ had transferred to another facility and $44 \%$ were LTFU Despite differences in the absolute proportions actoss the different studies, he recurning finding is that patients LTH make up the biggest share of patients not retained in ART programmes

\section{Reasons for patients LTFU in SSA}

Adherence to drug programmes is affected by the complex the drug itself $1^{15}$.care, Despite a paucity of qualitative research the drug itself . Despite a paucity of qualitative research
in developing countries, many reasons have been identified or hypothesized for the significant proportion of patients LTFU in ART programmes ${ }^{15}$. Several papers have identified financial constraints as a major cause of LTFU in SSA ${ }^{16}$. One study in Malawi found that $24 \%$ of patients originally recorded as LTFU, re-enrolled at the same site after ARTs were offered free of charge ${ }^{16}$. However, the variation in adherence rates in different areas of the country, irrespective of free access, suggests that other barriers to treatment are involved ${ }^{17}$. A qualitative study in Malawi's southern region of Chiradzulu found that fear and stigma were the main reason for $\mathrm{LTFU}^{18}$. Of the 221 patients interviewed, $74 \%$ of ART patients had not disclosed their HIV status to household members ${ }^{18}$. In addition, the only published systematic review examining the concerns of HIV patients enrolled on ART programmes also found that fear of disclosure was the most consistently cited barrier to treatment ${ }^{19}$. As well as discussing structural and socio-cultural issues, some research has concentrated on identifying which patient groups are most likely to become LTFU. Younger age at treatment initiation lower CD4 count at presentation 6 , type of ART regimen and the occurrence of side effects ${ }^{15}$ have all been found to be associated with LTFU in the early stages of treatment. Later on, a risk of LTFU arises from patients experiencing an improvement in health and subsequently assuming that treatment is no longer necessary; $28.3 \%$ of patients LTFU in rural Malawi cited improved health as their primary reason for leaving the programme ${ }^{18}$.

\section{$H I V$ and $A R T$ in Malawi}

Malawi's HIV prevalence is one of the highest in the world with the most recent Malawi Population-Based HIV Impact Assessment report estimating that $10.6 \%$ of the population (over 1 million people) are living with HIV ${ }^{22}$. The epidemic has affected all sectors of society, disproportionately argeting the youngest and most productive age groups, an of only 54.8 years $^{23}$. This burden is compounded by the of health workers; the ratio of 1 physician pe of twenty ${ }^{19}$. Nevertheless, substantial efforts to reduce the burden of HIV in Malawi have been made over the las decade, bringing about significant reductions in mortality, morbidity and transmission. New infections have declined from 98,000 in 2005 to 34,000 in 2013, gaining 1.4 millio life-years as well as a $67 \%$ reduction in mother-to-child transmission over the same period'. This has largely been attributed to remarkable increases in ART coverage across the country, with over 500,000 individuals initiated on ART since the introduction of free universal provision in 2004 Prior to this, ART was only available at a cost of U $\$ 25$ for a month's supply ${ }^{17}$. However, ART adherence is variable. In 2012, only 79\% of adults and children who started on ART in Malaw were taking them 12 months later, suggesting significant proportion of patients LTFU. National Reports in 2009, 2010 and 2012 have shown rates of LTFU of $12 \%$ $15 \%$ and $21 \%$ respectively, which indicate that the problem is increasing ${ }^{8,19}$ and with it, the impact on individuals. Patients missing visits in the first year of treatment have a two-fold increase in long-term mortality ${ }^{24}$. There is also a health ris to the general public posed by the likely increase in drug resistance ${ }^{25}$. In response to this, in 2014, Malawi launched the 2015-2020 National HIV/AIDS Strategic $\mathrm{Plan}^{26}$. A key objective involves "maintaining high levels of adherence and retention in ART programs"23. This study is a contribution to his objective; as the number and demographics of patient Tiv from ART at MMH was not known, it provides this insight into the frequency and easons patients may be LTFU locally.

\section{Study design}

This was a retrospective notes-based review.

\section{Setting}

MMH is a mission hospital located in southern Malawi, close to the border with Mozambique. The hospital serve catchment of 650,000 people who mainly live in rura largest part of the hospital's workload is in the assessment and treart of the hIV/AIDS, with 10,714 HIV tess do and 74,136 ART clinic attendances during 2015 alone 7 . The most recent prevalence study in the area found that $18 \%$ of . $10.3 \%$ ?

\section{Data collection}

using an existing clinical dataset, all patients on adult antiretroviral formulations at $\mathrm{MMH}$, who were classified as 'defaulters' over the previous 12 months (2nd quarter 2015 end of 1stquarter 2016), were included in the study. According to the local 2014 Malawian ART guidelines, defaulting was defined as "patients missing a scheduled follow- up visit by more than two months" ; a time frame that has recently been shown by Rachlis et al to represent the period $>0$ weeks) after which returning to clinic reduces substantially ${ }^{2}$. Data was collected from the patient cards stored in the outpatient ART clinic. The following variables were included in the analysis: gender, age, months on ART, time of year Lin clinical stage, ART regimen, reported side effects, number of doses missed in the previous 12 months, whether the patient has been followed up in the community if so, the length of time elapsed before follow up. To highlight comparisons amongst the studied population (the LTF group), a control group was identified and their data analysed. The control group was selected by using all of the 政 July 2016, and who had never defaulted.

\section{Statistical analysis}

Comparison data for LTFU and controls were analysed using the chi squared $\left(\chi^{2}\right)$ test, with alpha levels set at 0.05 .

Ethical considerations

Ethical approval was sought and approved by the MMH medical director and hospital board. Confidentiality was maintained by coding the patient ART cards anonymous patient identifiable information.

\section{Results}

In all, 136 patients had been LTFU over the previous 12 months at MMH. Of these, 43 patients had been missing ART cards, so the records of 93 patients LTFU were analysed. In the control group, 93 datasets were also analysed.
Table 1: Comparison data for LTFU and comparison group

\begin{tabular}{|c|c|c|c|}
\hline & & $\begin{array}{l}\text { Lost to Follow Up } \\
(n=93)\end{array}$ & Controls $(n=93)$ \\
\hline \multirow[t]{2}{*}{ Gender } & Male & $42(55 \%)$ & $28(30 \%)$ \\
\hline & Female & $51(45 \%)$ & $65(70 \%)$ \\
\hline \multirow[t]{5}{*}{ Age (years) } & $\leq 20$ & $3(3.2 \%)$ & $5(5.4 \%)$ \\
\hline & $21-30$ & $22(23.7 \%)$ & $13(14.0 \%)$ \\
\hline & $31-40$ & $36(38.7 \%)$ & $36(38.7 \%)$ \\
\hline & $41-50$ & $22(23.7 \%)$ & $23(24.7 \%)$ \\
\hline & $>50$ & $2(2.2 \%)$ & $7(7.5 \%)$ \\
\hline \multirow{4}{*}{$\begin{array}{l}\text { WHO } \\
\text { Clinical } \\
\text { Stage at } \\
\text { Initiation } \\
\text { of ART }\end{array}$} & 1 & $39(41.9 \%)$ & 47 (50.5\%) \\
\hline & 2 & $18(19.4 \%)$ & $15(16.1 \%)$ \\
\hline & 3 & $30(32.3 \%)$ & $26(28.0 \%)$ \\
\hline & 4 & $6(6.5 \%)$ & $5(5.3 \%)$ \\
\hline \multirow{5}{*}{$\begin{array}{l}\text { Duration } \\
\text { of } \\
\text { treatment } \\
\text { on ART } \\
\text { (months) }\end{array}$} & $0-12$ & $18(19.4 \%)$ & $10(10.8 \%)$ \\
\hline & $13-24$ & $20(21.5 \%)$ & $17(18.3 \%)$ \\
\hline & $25-36$ & $9(9.7 \%)$ & $11(11.8 \%)$ \\
\hline & $37-48$ & $18(19.4 \%)$ & $14(15.1 \%)$ \\
\hline & $>48$ & $28(30.1 \%)$ & $41(44.1 \%)$ \\
\hline \multirow{2}{*}{$\begin{array}{l}\text { Number } \\
\text { of Doses } \\
\text { of ART } \\
\text { missed } \\
\text { in last } 12 \\
\text { months }\end{array}$} & $0-3$ & $48(52 \%)$ & $61(66 \%)$ \\
\hline & $\geq 4$ & $45(48 \%)$ & $32(34 \%)$ \\
\hline
\end{tabular}

Patient demographics

There were a higher proportion of male patients in the LTFU group $(45.2 \%)$, when compared to the control group $(30.1 \%)(p=.0 .03)$. The mean age of patients LTFU was 35.7 ears, 2.9 years younger than the control group on average $\mathrm{p}=0.05)$

\section{WHO clinical stage at ART initiation}

There was no statistically significant difference in the clinica stages, using the WHO clinical staging system, between the $(\mathrm{p}=0.71)$.

The distribution of the total patients (136) LTFU across the year was as follows: 26 in the 2nd quarter of 2015, 29 in the 3rd quarter of 2015,49 in the 4th quarter of 2015 and 32 in he 1 st quarter of 2016

$A R T$-regimen and length since initiation of $A R T$

Of the patients who were LTFU, 94.6\% were taking ART dine and Efavirenz), the (in $2015^{24}$, and 2.2\% 
Number of Patients LTFU on ART in Each Quarter in $\mathrm{MMH}$

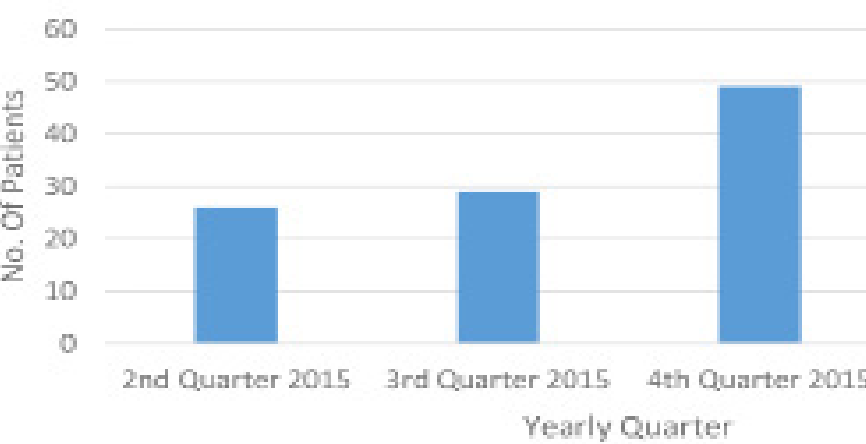

Figure 1: Number of Patients LTFU on ART in each quarter

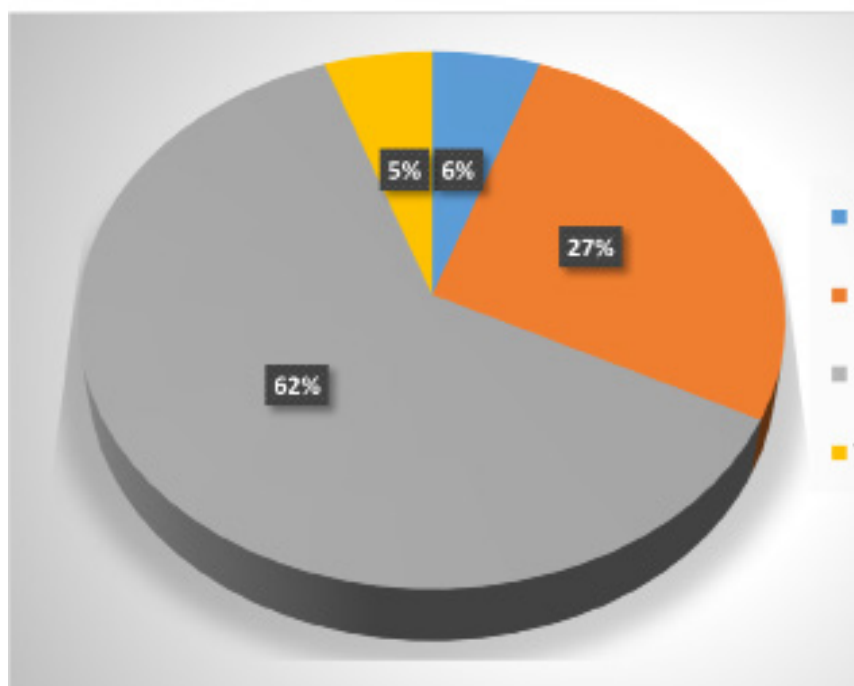

Figure 2: Reasons for patients becoming LTFU (Recorded by expert
clients) Pie Chart

had documented side effects in the previous 12 months (1 peripheral neuropathy and 1 hepatitis). The mean length of time on ART was 39.0 months in the LTFU group and 47.3 months in the control group, with a mean difference of 8.3 less months in the LTFU group $(\mathrm{p}=0.06)$.

The mean number of doses of ART missed in the previous 12 months was 13.15 in the LTFU group and 4.74 among the two groups, they were divided into those who had missed $0-3$ doses and those who had missed 4 or more doses, following the criterion used by the clinic for non-compliance. It was found that the proportion of patients missing more than 4 doses was higher among the LTFU group compared to the controls $(\mathrm{p}=0.05)$.

Tracing

Of the LTFU patients $34.4 \%$ had been traced in the community at the time of analysis, with a mean length of time between clinic non-attendance and tracing of 13 weeks, According to the expert clients tracing the patients in the $5.5 \%$ he $27 \%$ had moved to another area, $5.5 \%$ had died, documented in client files) and $62 \%$ gave no reason as to why they had missed appointments.

\section{Discussion}

The demographics of the MMH dataset echo previous findings in Malawi, in which male patients are more likely to get LTFU on $\mathrm{ART}^{27}$. The proportion of men in the LTFU group was $15.1 \%$ higher than in the control group, which was the only statistically significant finding of the study, with a chi-squared p-value of 0.03 . The reasons for this cannot be definitely ascertained, since no interview were undertaken with men who had been LTFU. However, previous studies in Malaw have shown that men are also less likely to know about their HIV status and to access treatment than women, suggesting that the gender disparity is one that encompasses al areas of HIV care ${ }^{17}$. The LTFU group also tended to be younger than the control group, with a mean difference of 3 years and a chsquared value of 0.06 . Fear of disclosure and stigma may be disproportionately affecting ounger men, but more qualitative research is required to explore this. What this study, a well as a 2017 retrospective cohort study by Tweya et al, does illuminate is that both men and younger patients are at risk of LTFU in Malawi ${ }^{28}$. Therefore, a recommendatio that interventions should be aimed secifically at men to improve their health outcomes.

\section{Time of year LTFU}

A higher proportion of those found LTFU were in the 4th quarter of 2015 and 1 st rainy season in Mulanie District, which hinders the ability of patients to travel over long distances. Although data over several years would be needed to confirm this trend, this is another matter warranting further research. Statistical confirmation Ild then be used to argue for more decentralised furtion of ART during the rainy season. This has been Neno District which found that ART decentralisation halved the average distance to health centres and was associated the average distance to health centres and was associated

\section{$A R T$-regimen and length since initiation of $A R T$}

Two of the most instructive findings from the study came from an examination of the relationship between the length of time on ART and the likelihood of being LTFU. The

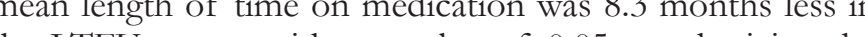
the LTF group, with a p-value of 0.05 , emphasizing the need for more intensive follow-up in the early stages of Patment, when patients are at the highest risk or LTHU. atsere more likely to get LTFU if they had missed 4 or more doses in the previous 12 months ( $\mathrm{p}$-value $=0.05$ ) of this represents another at-risk population who can be The pes and could be given support before they get LTFU. The presence of side effects was not predictive of whethe pate last would get LTFU, with only $2.2 \%$ reporting them in the last 12 months. This is significantly lower than the figures quoted in trials looking at the rates of side effects in the 5 A ART regimen, which is closer to $10 \%$. This is possibly related to a fallure in MMH documentation and so need further research, especially in the light of a recent multicountry qualitative study which found that every additional

\section{Tracing}

Despite operating an expert client system in the clinic, attempting to trace and retrieve patients who miss their medication refills or appointments before they are officially classified as "defaulters", only a third of those LTFU had received follow-up visits at the time of this study, suggesting that the community tracing is inconsistent. For those who were seen, the mean length of time between clinic nonattendance and tracing was 13 weeks. After taking into account the two-month delay that defines 'defaulting' in Malawi, this would mean a period of more than five months without medication for these patients, putting them at significant risk of adverse health consequences. When discussing this, one of the expert clients reported that a main barrier to seeing patients quickly was a lack of transport in the more remote areas, with their only bike currently broken and in need of servicing. Early follow-up has been shown to improve
retention, with the largest ART provider in central Malawi finding with the largest ART provider in central Malaw finding that following up patients within 3 weeks of a missed patients followed-up returned to clinic, this approach could paticnts follow be effective in

Another potential improvement to be made in the followup process is to take advantage of the growing number of mobile phone users in the developing world, with a project in Kenya finding that phone contacting improved ART adherence rates ${ }^{32}$. The expert clients also said that using phones would make for a more efficient follow-up. However, they were not currently provided with phone credit; so again this would need more funding to be made available.

\section{Limitations}

A significant limitation of this study was a reliance on the routine notes provided by the clinic, which often had large gaps and contained inconsistencies. The 43 initial missing records represent a potential selection bias if there are systematic differences between these patients and those analysed. Even when patient ART cards were available, some of the data was not entered: 8 ages were not recorded and for many patients, only their year of birth (not date) was documented. Absence of accurate data could have been source of bias in age-related analyses.

Furthermore, there was no follow-up of two thirds of the Futhe pre the was no follow-up two the of the quantity. Without knowing if these patients had died, had moved elsewhere or had actively decided to stop treatment, patients who were followed up $62 \%$ were documented having "no reason" for leaving the programme. As their reasons are clearly more complex than that these patients need to be interviewed in more depth to see whether they can be returned to the ART programme, with a view to reducing both morbidity and mortality. Ultimately the biggest limitation was the study desion; a case-control study desion would be ideal. The way the control group was selected, which was by selecting patients attending over the course of a week, may not be truly comparable to the LTFU group, which was selected over the course of a year. This method was chosen as it was least distuptive to the running of the clinic in the short time available for data collection. However, as the control group was was not randomly Conclusion

The global response to the challenge of scaling up access to ART in the developing world signifies one of the largest public health successes in recent history ${ }^{6}$. However, high rates of LTFU have the potential to reverse the gains that have been achieved thus far. Therefore, a focus of public health campaigns needs to be on long-term retention of patients on ART. The fallure of patients to comply with their treatment should not be accepted as inevitable, but as a health injustice, affecting the poorest and most marginalized sectors of HIV patients and invariably worsening their health outcomes. This study at Mulanje Mission Hospital Nalawi, has highlighted the importance of comprehensive facility-level data-collection, both to identify local patien populations at risk of becoming LTFU and to assess the follow-up measures in place to bring these LFTU patients back into the programme. The authors found evidence that patients most likely to get LTFU in MMH were young men, over 4 doses in the last 12 months. The increse in pare ITFU during the 1st and 4th quaters of the year need further investigations and may highlight a need to provide the during these times. There was alo evidence of selios dine follow-up process, some of which could (a) celits. In the immediste future, there is an ungent need to understand why patients are ITFU in MMH, to deve argeted interventions to prevent it. In order to further understanding, it is proposed that a qualitative study using semi-structured interviews with LTFU patients should be planned and undertaken in the area.

\section{References}

. LeVasseur M. T., Goldstein N. D., Welles S. A public health perspective on HIV/AIDS in Africa: Victories and unmet challenges. pathophys.2014.07.001.

2. UN. The Millennium Deve
Nations, New York; 2010

2010 Developer

3. Rasschaert F., Koole O., Zachariah R., Lynen L., Manzi M., Van Damme W. Short and long term retention in antiretroviral care in
health facilities in rural Malawi and Zimbabwe. BMC Health Serv Res 2012;12(1);1. doi: https://doi.org/10.1186/1472-6963-12-444

4. Rosen S., Fox M. P., Gill C. J. Patient retention in antiretroviral herapy programs in sub-Saharan Africa: a systematic review. PLoS

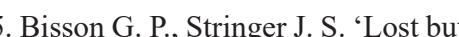

S. improving patient retention in AIDS treatment
2009 6(10) doi: 10.1371/journal.pmed.1000174

6. Fox M. P., Rosen S. Patient retention in antiretroviral therapy programs up to three years on treatment in sub-Saharan Africa, 2007 10.1111/j.1365-3156.2010.02508.x

7. Shakespeare, R. Malawi: Challenges For Health [Lecture to University of Bristol SSC students]. Mulanje Mission Hospital. 8 July 016.

8. Joint United Nations Programme on HIV/AIDS (UNAIDS). (2014) The Gap Report.' Geneva: UNAIDS.

9. Bisson G. P., Gaolathe T., Gross R., Rollins C., Bellamy S., Mogorosì 
M. 'Overestimates of survival after HAART: implications for global scale-up efforts.' PloS Medicine. 2008;3(3). doi: 10.1371/journal. pone. 0001725

10. Bassett I. V., Wang B., Chetty S., Mazibuko M., Bearnot B., Giddy J. Loss to care and death before antiretroviral therapy in Durban, South Africa. J Acquir Immune Defic Syndr. 2009; 51(2):135-9

11. Geng E. H., Emenyonu N., Bwana M. B., Glidden D. V., Martin J. N. Sampling-based approach to determining outcomes of patients lost to follow-up in antiretroviral therapy scale-up programs in Africa. JAMA. 2008;300(5):506-507. doi: 10.1001/jama.300.5.506

12. Zachariah R., Harries A. D., Srinath S., Ram S. 'Language in tuberculosis services: can we change to patient-centred terminology and stop the paradigm of blaming the patients?' Int J Tuberc Lung Dis. 2012;16(6): 714-717. doi: 10.5588/ijtld.11.0635.

13. Dalal R. P., MacPhail C., Mqhayi M., Wing J., Feldman C., Chersich M. F. Characteristics and outcomes of adult patients lost to follow-up at an antiretroviral treatment clinic in Johannesburg, South Africa. J Acquir Immune Defic Syndr. 2008; 47(1):101-107. doi: 10.1097/ QAI.0b013e31815b833a

14. Krebs D. W., Chi B. H., Mulenga, Y., Morris M., Cantrell R. A., Mulenga L. etal. Community-based follow-up for late patients enrolled in a district-wide programme for antiretroviral therapy in Lusaka, Zambia. AIDS care. 2008;20(3):311-317. doi: 10.1080/09540120701594776.

15. Miller C. M., Ketlhapile M., Rybasack-Smith H., Rosen S. Why are antiretroviral treatment patients lost to follow-up? A qualitative study from South Africa. Trop Med Int Health. 2010;15(s1):48-54. doi:10.1111/j.1365-3156.2010.02514.x.

16. Hosseinipour M. C., Neuhann F. H., Kanyama C. Lessons Learned From a Paying Antiretroviral Therapy Service in the Public Health Sector at Kamuzu Central Hospital, Malawi 1-Year Experience. J Int Assoc Provid AIDS Care. 2006;5(3):103-108. doi: $10.1177 / 1545109706288722$

17. Muula A. S., Kataika E. (2008) Assessment of equity in the uptake of anti-retrovirals in Malawi. EQUINET Discussino Paper 58. University of Malawi, College of Medicine, Department of Community Health. [Online]. Available at: https://www.researchgate. net/publication/255594360_Assessment_of_equity_in_the_uptake_of anti-retrovirals_in_Malawi (Accessed 18th March 2018)

18. McGuire M., Munyenyembe T., Szumilin E., Heinzelmann A., Le Paih M., Bouithy N. et al. Vital status of pre-ART and ART patients defaulting from care in rural Malawi. Trop Med Int Health. 2010;15(s1):55-62. doi: 10.1111/j.1365-3156.2010.02504.x.

19. Rachlis B. S. Losses to Follow-up from an Antiretroviral Therapy (ART) Program in the Zomba District of Malawi. 2013. Doctoral dissertation, University of Toronto.

20. Chan A. K., Mateyu G., Jahn A., Schouten E., Arora P. Outcome assessment of decentralization of antiretroviral therapy provision in a rural district of Malawi using an integrated primary care model. Trop Med Int Health. 2010;15(s1): 90-97. doi: 10.1111/j.13653156.2010.02503.x.

21. Yiannoutsos C. T., Frangakis C. E., Musick, B. S. Samplingbased approaches to improve estimation of mortality among patient dropouts: experience from a large PEPFAR-funded program in Western
Kenya. PloS one. 2008;3(12). doi: https://doi.org/10.1371/journal. pone. 0003843

22. Ministry of Health, Malawi (2016) 'Summary Sheet Preliminary Findings: The Malawi Population-Based HIV Impact Assessment'

23. Ministry of Health, Malawi (2015) 'Malawi AIDS Response Progress Report.'

24. Mugavero M. J., Lin H. Y., Willig J. H., Westfall A. O., Ulett K. B. Missed visits and mortality among patients establishing initial outpatient HIV treatment. Clin. Infect. Dis. 2009;48(2):248-256. doi: $10.1086 / 595705$.

25. Brennan, A. T., Maskew, M., Sanne, I., Fox, M. P. The importance of clinic attendance in the first six months on antiretroviral treatment: a retrospective analysis at a large public sector HIV clinic in South Africa. J Int AIDS Soc. 2010;13(1): 49. doi: 10.1186/1758-2652-13-49

26. Ministry of Health, Malawi (2014) 'Clinical Management of HIV in Children and Adults.'

27. Rachlis B., Ahmad F., van Lettow M., Muula A.S., Semba M., Cole D.C. Using concept mapping to explore why patients become lost to follow up from an antiretroviral therapy program in the Zomba District of Malawi. BMC health services research. 2013;13(1):210. doi: 10.1186/1472-6963-13-210.

28. Tweya H., Feldacker C., Heller T., Gugsa S, Ng'ambi W, Nthala O. et al. Characteristics and outcomes of older HIV-infected patients receiving antiretroviral therapy in Malawi: A retrospective observation cohort study. PLoS One. 2017;12(7) doi: https://doi.org/10.1371/ journal.pone.0180232

29. Bilinski A., Birru E., Peckarsky M., Herce M., Kalanga N., Neumann C., Bronson G et al. Distance to care, enrollment and loss to follow-up of HIV patients during decentralization of antiretroviral therapy in Neno District, Malawi: A retrospective cohort study. PloS one. 2017;12(10). doi: https://doi.org/10.1371/journal.pone.0185699

30. Renju J., Moshabela M., McLean E., Ddaaki W., Skovdal M., Odongo F. et al. 'Side effects' are 'central effects' that challenge retention in HIV treatment programmes in six sub-Saharan African countries: a multicountry qualitative study. Sex Transm Infect. 2017. doi: 10.1136/sextrans-2016-052971.

31. Tweya H., Gareta D., Chagwera F. Early active follow-up of patients on antiretroviral therapy (ART) who are lost to follow-up: the 'Back-to-Care'project in Lilongwe, Malawi. Trop Med Int Health.. 2010;15(s1):82-89. doi: 10.1111/j.1365-3156.2010.02509.x.

32. Lester R. T. Effects of a mobile phone short message service on antiretroviral treatment adherence in Kenya (WelTel Kenya1): a randomised trial. Lancet. 2010;376(9755) :1838-1845. doi: https://doi. org/10.1016/S0140-6736(10)61997-6

33. Mberi M. N., Kuonza L. R., Dube N. M., Nattey C., Manda S. Determinants of loss to follow-up in patients on antiretroviral treatment, South Africa, 2004-2012: a cohort study. BMC health services research. 2015;15(1):1. doi: 10.1186/s12913-015-0912-2. 